\title{
Semantic and episodic processes differently predict false memories in the DRM task
}

\author{
Daniele Gatti ${ }^{1 *}$, Luca Rinaldi ${ }^{1,2}$, Giuliana Mazzoni ${ }^{3,4}, \&$ Tomaso Vecchi ${ }^{1,2}$ \\ ${ }^{1}$ Department of Brain and Behavioral Sciences, University of Pavia, Pavia, Italy \\ ${ }^{2}$ Cognitive Psychology Unit, IRCCS Mondino Foundation, Pavia, Italy \\ ${ }^{3}$ Faculty of Medicine and Psychology, University La Sapienza, Rome, Italy \\ ${ }^{4}$ School of Life Sciences, University of Hull, Hull, United Kingdom
}

* Corresponding author:

Daniele Gatti, Department of Brain and Behavioural Science, University of Pavia, Piazza Botta 6, 27100 Pavia, Italy. e-mail address: daniele.gatti@unipv.it 


\begin{abstract}
There is a fervent debate about the processes underpinning false memories formation. Seminal theories have suggested that semantic memory would be involved in false memories production, while episodic memory would counter their formation. Yet, direct evidence corroborating such view is still missing. Here, we tested this possibility by asking participants to perform the Deese-RoedigerMcDermott (DRM) task, a typical false memory paradigm, in which they had to study lists of words and subsequently to recognize and distinguish them from new words (i.e., the false memory items). The same participants were also required to perform a semantic task and an episodic-source memory task. Our results showed that a higher number of false memories in the DRM task occurred for those participants with better semantic memory abilities, while a lower number of false memories occurred for participants with better episodic abilities. These findings support a key role of semantic processes in false memory formation and, more generally, help clarify the specific contribution of different memory systems to false recognitions.
\end{abstract}

\title{
Keywords:
}

False memory, DRM, episodic memory, semantic memory, individual differences 


\section{Introduction}

Human memory cannot be simply conceived as a recorder that passively encodes, stores and faithfully retrieves information, but rather as a system that actively reconstructs information from memory traces (Schacter, 2001; Vecchi \& Gatti, 2020). Indeed, beyond accurate remembering, it has been shown that humans use their semantic memory to encode, store and remember information adapting it to their own previous knowledge, favoring consequently a gist extraction: in such a process, memory distortions and false memories may thus occur (Bartlett, 1932; Sulin \& Dooling, 1974).

One of the most widely used task to create false memories is the Deese-Roediger-McDermott task (DRM; Deese, 1959; Roediger \& McDermott, 1995). During the DRM task, participants are first asked to encode several words divided in lists (i.e., within each list, the words are semantically/associatively related to a non-shown target word, named critical lure; e.g., table, sit, legs, seat, couch, desk, etc. - critical lure: chair) and then, after a brief distracting task, participants are asked to perform a recognition task (i.e., they have to indicate whether a given word was part of the memorized lists or not). Typically, during the recognition task, participants report as "old" (i.e., as previously memorized) a fairly large number of critical lures, although these words have not been presented before (for a review see, Gallo 2010).

The two main theories proposed to explain participants' performance in the DRM task have traced back false memories occurrence to semantic processing. In particular, according to the activationmonitoring framework (AMF - Gallo \& Roediger, 2002; Roediger, Watson, McDermott, \& Gallo, 2001), the critical lure would be associatively hyperactivated by the presentation of the studied words (i.e., spreading activation), leading to high levels of false recognitions. Alternatively, according to the fuzzy-trace theory (FTT - Brainerd, \& Reyna, 2002; Reyna \& Brainerd, 1995), participants would encode two different memory traces: a trace linked to episodic and perceptive features of the studied items, called verbatim trace, and a trace linked to the semantic content of each list, called gist trace, which would be responsible for the production of the false memories. Besides predicting a semantic basis for false memory production, both theories also predict that adequate source or episodic memory processes can reduce the occurrence false recognitions. That is, according to the AMF, if participants can successfully distinguish between words presented and words hyperactivated but not presented, the production of false memories would decrease (Gallo, 2010). Alternatively, according to the FTT, the verbatim trace would be involved in the correct rejection of non-presented words, since no episodic memory trace is available for those stimuli (Brainerd, \& Reyna, 2002; Reyna \& Brainerd, 1995).

In line with these theoretical perspectives, it has been shown that false memory occurrence can be predicted on a semantic and associative basis (Brainerd, Yang, Reyna, Howe, \& Mills, 2008; 
Roediger et al., 2001). In particular, the backward associative strength (BAS, i.e., the association strength from the words that compose each list to the critical lure) is thought to be the central factor in determining false recall and false recognition (Roediger et al., 2001). Furthermore, recent studies also showed that the semantic component that underlies false memories can be decomposed into various sub-components (Cann, McRae, \& Katz, 2011) and that higher false recognition rates occur for new words with higher semantic similarity with the ones studied (Gatti, Rinaldi, Marelli, Mazzoni, \& Vecchi, 2022; Gatti, Marelli, Mazzoni, Vecchi \& Rinaldi, 2022). At the same time, it has been shown that inducing a higher monitoring process warning participant about the false memory effect in the DRM (Gallo, Roberts, \& Seamon, 1997) or instructing them to focus on the distinctive aspects of each presented word (Westerberg \& Marsolek, 2006) would reduce false recognitions, thus pointing to the possible opponent role of episodic memory processes in countering memory distortions. Yet, the experimental manipulations introduced by prior research do not fully clarify the extent to which semantic and episodic memory processes differently contribute to false memories.

Previous studies have been indeed able to predict participants' memory performance in the DRM task (e.g., Roediger et al., 2001), but this evidence is mainly limited to semantic processing and based only on manipulations at the item level (i.e., the type of stimuli used). Here, to directly probe the role of semantic and episodic processes in memory distortions, we adopted an individual differences approach. An advantage of an individual differences framework is that it allows to conceive individuals along continuous dimensions reflecting their semantic and episodic memory abilities, and to relate such natural variabilities with false memories production. Notably, such an approach has proven to be successful in demonstrating that that higher working memory abilities (Holden, Conway, \& Goodwin, 2020; Unsworth \& Brewer, 2010; Watson, Bunting, Poole, \& Conway, 2005) or high memory self-efficacy (Iacullo, Marucci, \& Mazzoni, 2016) are associated with a lower occurrence of false memories. Similarly, age differences have been linked to false memories, with false memories increasing in older individuals (e.g., Balota et al., 1999; Norman \& Schacter, 1997; Tun, Wingfield, Rosen, \& Blanchard, 1998; Watson, Balota, \& Sergent-Marshall, 2001; but cfr. also: Pansuwan et al., 2020). Moreover, other studies investigating the link between creativity abilities such as convergent and divergent thinking have shown that the former, but not the latter, is associated with increased false memories (Dewhurst, Thorley, Hammond, \& Ormerod, 2011). Finally, individual differences in need for cognition, an index that characterizes individuals' preferences for engaging in effortful information processing (Cacioppo \& Petty, 1982), can predict memory performance in the DRM task: in particular, participants with a high need for cognition, thus more likely to engage in effortful information processing, typically show a greater occurrence of false recalls (Leding, 2011) and false recognitions (Graham, 2007). 
Here, following the main theoretical accounts linking false memories with semantic and episodic processes, we aim to systematically dissociate the possible contribution of each memory system to memory distortions. We thus expect participants' false recognitions of the critical lures to be positively associated with their semantic abilities (i.e., higher number of false memories for individuals with better semantic memory abilities) and negatively related with their episodic abilities (i.e., lower number of false memories for individuals with better episodic memory abilities). In addition to this, since the FTT posits that the verbatim trace is linked to both episodic and perceptive features of the studied items (Brainerd, \& Reyna, 2002; Reyna \& Brainerd, 1995), we also investigated participants' source memory abilities. Participants were asked to perform a semantic task and an episodic-source memory task, from which we extracted the relative memory scores. We then predicted false and veridical memories occurrence in the DRM task using the scores extracted.

\section{Methods}

\section{Participants}

Sixty-four Italian right-handed students (23 males, $M$ age $=23.89$ years, $S D=3.18)$ participated in the study. All participants were native Italian speakers, had normal or corrected to normal vision and were naïve to the purpose of the study. Informed consent was obtained from all participants before the experiment. The protocol was approved by the psychological ethical committee of the University of Pavia and participants were treated in accordance with the Declaration of Helsinki.

\section{Stimuli and procedure}

Participants were tested online using Psychopy (Pierce, 2007, 2009; Pierce \& MacAskill, 2018; Pierce et al., 2019) through the online platform Pavlovia (https://pavlovia.org/). Participants performed the three tasks in three different days (i.e., the three sessions had to be completed within 1 week) at the same time of the day. The order of the tasks was counterbalanced across participants.

\section{Semantic memory task}

The task used was an associative/semantic priming task, in which participants were shown two stimuli (i.e., a prime stimulus and a target one, sequentially presented on the screen) and then were asked to judge if the second one (i.e., the target) was a word or a pseudoword. Generally, participants reaction times tend to be predicted by the degree of semantic relationship between prime and target words, with faster reaction times occurring for more related pairs. This speeding up is thought to reflect the enhanced involvement of semantic processing (Hutchison et al., 2013). 
Primes were 120 words selected from the Italian database provided by Montefinese and colleagues (Montefinese, Ambrosini, Fairfield, \& Mammarella, 2013). For each prime, a target word was chosen using the distributional semantic model SNAUT (Mandera, Keuleers, \& Brysbaert, 2017; Marelli, 2017; http://meshugga.ugent.be/snaut-italian/), by selecting among the most semantically similar words and avoiding repetitions of the same target word across the task (for a review on distributional semantic models, see: Günther, Rinaldi, \& Marelli, 2019). Distributional semantic models have been shown to be high-performing across a wide range of semantic tasks (e.g., Baroni et al., 2014; and see also Gatti, Marelli, \& Rinaldi, in press), and they are equivalent to psychologically grounded associative learning models (Günther et al., 2019; Mandera et al., 2017). Critically, the semantic similarity index extracted from these databases is thought to involve both associative and semantic processes (for a similar approach with the DRM task, see Gatti et al., 2022a, 2022b). Then, the 120 word-pairs obtained were divided into two sets of 60 word-pairs (i.e., related and unrelated). In the unrelated set, primes and targets were pseudo-randomly mixed in order to remove the semantic link between the words (i.e., characterizing in turn the related set). From the same Italian database 120 additional words were extracted and transformed into pseudowords (i.e., reversing two letters: paper - pepar). Pseudowords were readable, but meaningless.

Each prime appeared twice, one time followed by a word, and another time by a pseudoword. Related and unrelated pairs were comparable in terms of length and logarithm of the frequency of the first and second word, as well as in total length of the two words paired together and their paired logarithm of the frequency (all $p \mathrm{~s}>.44$, all BFs $<.24$ ).

Participants were shown two letter strings stimuli presented sequentially one after the other, and were required to judge if the second stimulus was a word or not. Participants were instructed to silently read the first letter string and to respond only to the second one as fast and as accurately as possible by pressing the left/right key (A and L) using the left and right index fingers, respectively; the response keys were counterbalanced across participants. The trials were shown in random order.

Each trial started with a central fixation cross (presented for $500 \mathrm{~ms}$ ) and was followed by a first word (presented for $200 \mathrm{~ms}$ ) and then by a second word (presented for $500 \mathrm{~ms}$ ). Participants' response ended the trial and moved to the fixation cross of the next trial. See Figure 1 for a schematic representation of the semantic memory task. 
Semantic memory task
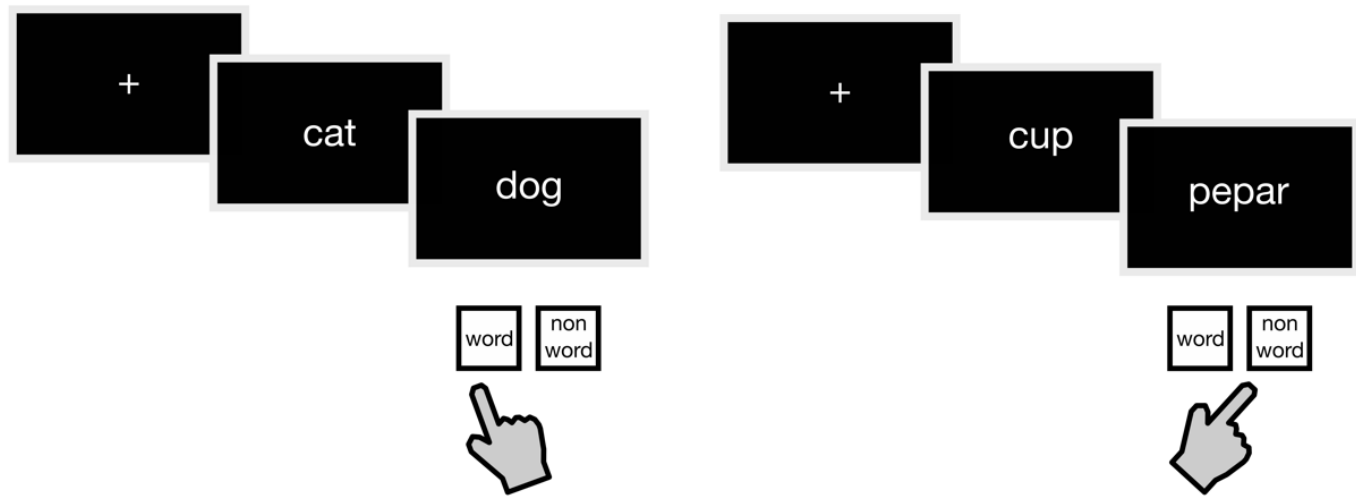

\section{Episodic-source memory task}

Encoding

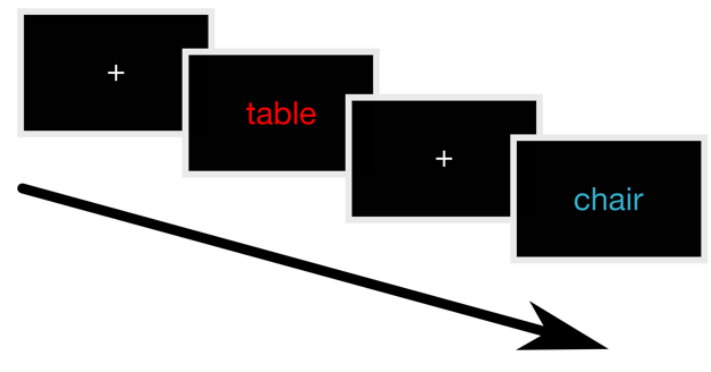

DRM task

Encoding

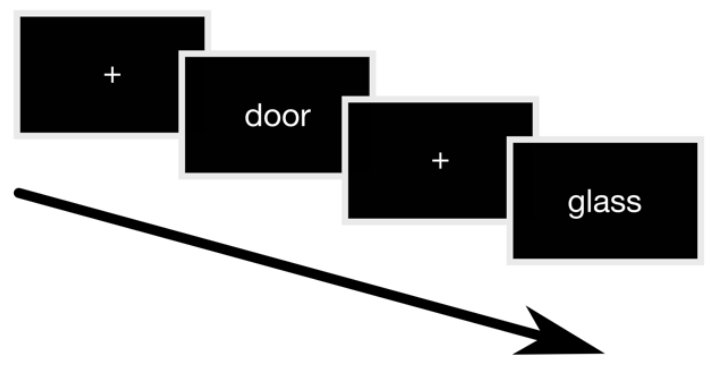

Recognition

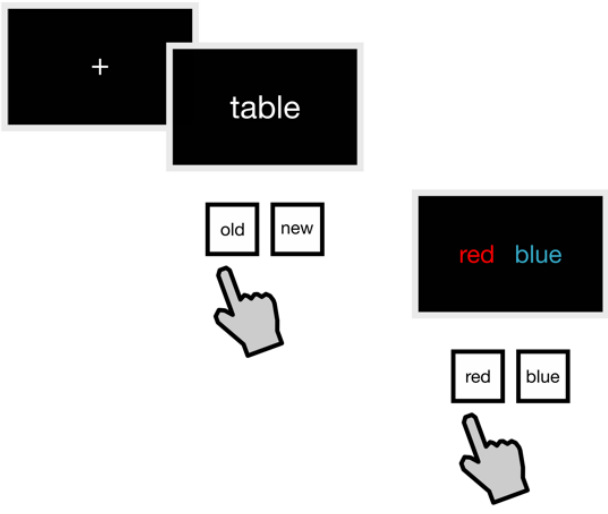

Recognition

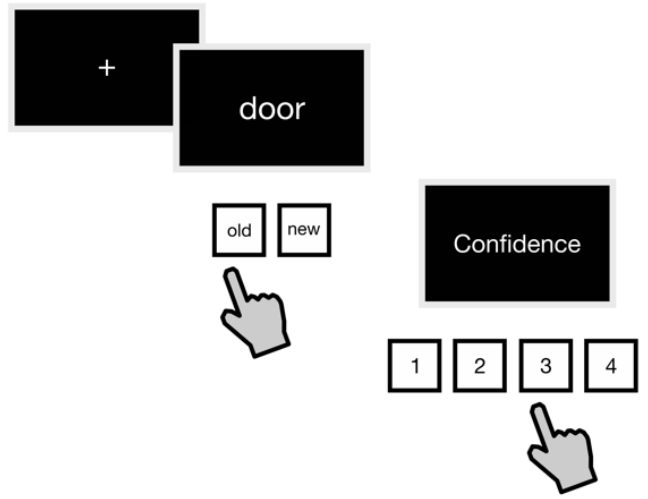

Figure 1. Schematic representation of the semantic priming task used to measure participants' semantic abilities (top), of the task used to measure participants' episodic and source memory abilities (middle), and of the two phases of the DRM task (bottom). 


\section{Episodic-source memory task}

We used an episodic-source memory task similar to the one recently employed by Chen and colleagues (Chen, Lo, Liu, \& Cheng, 2016). In a first phase, participants were asked to study several words printed in different colors and then, after a distracting task, to discriminate old/new words and to retrieve the color in which they have been originally shown. This task is thought to reflect general episodic abilities (i.e., the old/new judgements) and deeper source-memory processing (i.e., the color recognition).

We selected 100 concrete words from the Italian database provided by Della Rosa and colleagues (Della Rosa, Catricalà, Vigliocco, \& Cappa, 2010). The 100 words were divided into two sets of 50 words. The first 50 were divided into two subsets, presented respectively in red and blue fonts. These words were presented during the memorization phase and were therefore "old" items in the subsequent recognition task. The remaining 50 words were not shown in the study phase and were used as "new" items in the subsequent recognition phase.

The two sets (i.e., as well as the two subsets) were comparable in terms of concreteness, imageability, familiarity, age of acquisition, context availability, abstractness, mode of acquisition, number of letters and logarithm of the frequency (all $p \mathrm{~s}>.32$, all $\mathrm{BFs}<.33$ ).

During the first part of the task (i.e., encoding phase), participants were instructed to memorize 50 words: 25 words were colored in red and 25 words were colored in blue. Each trial started with a central fixation cross (presented for $500 \mathrm{~ms}$ ) followed by a word (presented for $1500 \mathrm{~ms}$ ) presented in either red or blue, followed by a blank screen (presented for $300 \mathrm{~ms}$ ), then the script moved automatically to the next fixation cross. The words were shown in random order. At the end of the study phase, participants were required to perform an attention task (i.e., a modified version of the go-no go task) as a brief distracting task for 2 minutes.

Then participants had to complete the recognition phase. Participants were instructed to make old/new judgments and to respond as fast and as accurately as possible by pressing the left/right keys (A and L); the response keys were counterbalanced across participants. In case of "old" judgements (i.e., when participants judged the word as already presented in the memorization phase), the script moved to the next screen showing two question marks (one in the left side and one in the right side, one in red and the other in blue, with the position counterbalanced across participants); the participants were asked to make a source judgment by identifying the correct color in which the word was presented in the memorization phase. On the contrary, in case of a "new" judgement, participants were instructed to press the space bar, then the script moved automatically to the next trial. The trials were shown in random order. In this phase, each trial started with a central fixation cross (presented for $1000 \mathrm{~ms}$ ) 
followed by a word (presented for $2500 \mathrm{~ms}$ ) and, after participants' response, by the source judgement. See Figure 1 for a schematic representation of the episodic-source memory task.

\section{DRM task}

We used a typical version of the DRM task (Roediger \& McDermott, 1995). In a first phase, participants were asked to memorize several words grouped by lists (within each list, the words were semantically/associatively related to a non-shown target word, named critical lure; see below) and then, after a distracting task, to discriminate old/new words. Typically, participants report as "old" (i.e., as previously memorized) a large number of critical lures, although they were not in the studied items (for a review see, Gallo 2010).

We selected 10 lists of words from the normative data for Italian DRM reported by Iacullo and Marucci (2016). Each list was composed by 15 words associatively related to a non-shown word (called critical lure). In this study, the words in position 13 and 14 were excluded and used as weakly related lures during the recognition phase, while the $15^{\text {th }}$ word was not used; thus, participants studied 12 words lists. From the remaining lists reported by Iacullo and Marucci (2016) - the ones not used in the present study - we extracted the words used as control stimuli.

The recognition phase was composed of 80 words, half of which had been studied and half of which had not. The 40 studied words presented in the recognition phase were those in positions 1, 4, 7 and 10 in the studied lists. Of the 40 non-studied words, 10 were the critical lures of the studied lists, 20 were weakly related lures, and 10 were unrelated words. The unrelated words were chosen randomly from the above-mentioned excluded lists. For example, for the list with slow as critical lure, the words included in the list were: fast, snail, trend, dance, train, adagio, elderly, calm, delay, waltz, clear and tortoise; the words used in the recognition phase as studied words were: fast, dance, elderly and waltz; the words used as weakly related lures were peaceful and ant; the unrelated word was lemon.

During the first part of the task, participants had to memorize a series of words (i.e., they were required to study 10 lists of words without interruptions). Participants were shown the 12 words that composed each of the 10 lists in descending forward associative strength (FAS; i.e., the association strength from the critical lure to the word that compose the list). The order by which the lists were presented was random, while the order of the words within each list was fixed (see Roediger \& McDermott, 1995). Each trial started with a central fixation cross (presented for $500 \mathrm{~ms}$ ) followed by a word (presented for $1500 \mathrm{~ms}$ ) and a blank screen (presented for $300 \mathrm{~ms}$ ), then the script moved automatically to the next fixation cross. 
At the end of the encoding phase, participants were required to perform an attention task (i.e., a modified version of the go-no go, different from the one employed during the episodic task) as a distracting task for 2 minutes.

Then participants were asked to perform the recognition phase. Participants were instructed to make old/new judgments and to respond as fast and accurately as possible by pressing the left/right key (A and L) using both hands; the response keys were counterbalanced across participants. After the old/new judgment, the script moved to the next screen and participants were asked to make a confidence judgement about their response using the keys 1, 2, 3, and 4 (i.e., with the key 1 representing the lowest levels of confidence and the key 4 representing the highest levels of confidence). The trials were shown in random order.

Each trial started with a central fixation cross (presented for $1000 \mathrm{~ms}$ ) followed by a word (presented for $2500 \mathrm{~ms}$ ) and then by the confidence judgement. The confidence judgement ended the trial and the fixation cross of the next trial was presented. See Figure 1 for a schematic representation of the DRM task.

\section{Computation of the memory components scores}

Signal detection theory measures were calculated using R-Studio (RStudio Team, 2015), by means of the psycho package (Makowski, 2018).

The semantic memory score was computed as the priming effect z-transformed score (i.e., in order to get homogeneous values from different memory scores used as predictors in the final analyses) induced by the prime on the processing of the target word (i.e., the speeding up in participants' correct reaction times; RTs). That is, for each participant, the semantic score was computed by subtracting the mean RTs for related prime-target words pairs to the mean RTs for unrelated prime-target words pairs (i.e., positive values indicate a facilitation in the processing of the target word as induced by the related prime as compared to the unrelated prime and, consequently, a stronger activation of the semantic memory network). In analogy with previous studies using associative and semantic priming tasks (Argyropoulos \& Muggleton, 2013), we analyzed responses with RTs $<1200 \mathrm{~ms}$ (5\% of the trials excluded), since slower responses are thought to reflect distraction or low familiarity with the words showed, rather than lexical access (Perea \& Gotor, 1997). Similarly, responses judged too fast (RTs $<300 \mathrm{~ms}$ ) were excluded ( $2 \%$ of the trials excluded). Critically, and as expected, participants' RTs for related words were overall significantly faster compared to RTs for unrelated words, $t(63)=$ $2.36, p=.02$, thus reflecting a facilitation due to semantic relatedness.

The episodic memory score was computed as participants' $d$ ' (Stanislaw \& Todorov, 1999), that is, the z-value of the hit-rate ("yes" response when the correct response is "yes") minus the z-value of 
the false-alarm rate ("yes" response when the correct response is "no") in old/new judgements of the episodic task. Participants' d' was computed only on responses with RTs $>300 \mathrm{~ms}$ and RTs $<3000$ $\mathrm{ms}(6 \%$ of the trials excluded).

The source memory score was computed as participants' $d$ ' in the source judgment of the episodic task. In this case, we considered only trials in which participants responded "old" to old words (i.e., only trials in which there was actually a color source to be accessed to), since including also the other items would have caused an overestimation in participants' error rate. To compute the source memory score, we considered as hits those trials in which participants correctly identified the correct color, while we considered as false alarms those in which participants indicated the wrong color. We included only trials with RTs $>150 \mathrm{~ms}$ and RTs $<3000 \mathrm{~ms}$ ( $2 \%$ of the trials excluded).

The semantic memory score was not correlated with the episodic memory score, $r=-.09, p=.46$, nor with the source memory score, $r=-.11, p=.39$. The correlation between episodic memory score and the source memory score was higher but not significant, $r=.23, p=.07$. The correlation between episodic and source memory scores is consistent with the theoretical view according to which they would represent two sub-components of the same memory system (Baddeley, Eysenck, \& Anderson, 2009).

Finally, in the DRM task, trials with RTs $<300 \mathrm{~ms}$ and RTs $>3000 \mathrm{~ms}$ were excluded from the analyses ( $4 \%$ of the trials excluded for old/new judgements; $11 \%$ of the trials excluded for confidence judgements). The analyses on confidence judgements were performed including only the trials in which participants responded "old" (i.e., hits for studied words and false alarms for critical lures, weakly related lures and unrelated words).

\section{Data analysis}

All the analyses were performed using R-Studio (RStudio Team, 2015). Data were analyzed through a mixed-effects approach, which incorporate both fixed-effects and random-effects (i.e., associated to statistical units as participants and task stimuli) and provide more detailed information about relationships among predictors and outcome variables compared with Pearson correlation (which simply measures the strength of the linear relationship between each selected pair of variables independent of the others; Koerner \& Zhang, 2017). Generalized linear mixed models (GLMMs) were run using the lme 4 package (Bates, Maechler, Bolker, \& Walker, 2015), while cumulative link mixed models (CLMMs) were run using the ordinal package (Christensen, 2019). The graphs reported were obtained using the effects package (Fox, 2003; Fox \& Weisberg, 2019).

First, we explored the memory processes subserving false and veridical memory. We ran a GLMM having old/new judgements in the DRM task (i.e., "new" responses were scored as 0, whereas "old" 
responses as 1) as the dependent variable and subjects and items as random intercepts. In particular, in our statistical model, we additively included as predictors the z-transformed semantic, episodic and source memory scores along with their interaction with the Type of stimuli (critical lures vs. weakly related lures vs. unrelated words vs. studied words). That is, in the lme 4 syntax, we tested the following model:

$$
\text { Response } \sim(\text { Semantic }+ \text { Episodic }+ \text { Source }) * \text { Type }+(1 \mid \text { Participant })+(1 \mid \text { Item })
$$

Second, we explored the memory processes subserving confidence judgements when making veridical and false memories. We ran a CLMM with confidence judgements (i.e., from low to high confidence, 1 vs. 2 vs. 3 vs. 4 , as a factor) as the dependent variable and subjects and items as random intercepts. The semantic, episodic and source memory scores were additively included along with their interaction with the type of stimuli (critical lures vs. weakly related lures vs. unrelated words vs. studied words; i.e., the model is analogous to the one reported above for old/new judgements). In the Results section we report only the analyses on old/new judgements, since no significant effect was found for confidence judgements (i.e., the results on confidence judgements are reported as Supplementary Materials).

\section{Results}

The Pseudo- $R^{2}$ (total) of the model estimated was $=.46$ and the $P$ seudo- $R^{2}$ (marginal) was $=.32$. The effects of episodic memory score, $\chi^{2}(1)=5.41, p=.02$, semantic memory score, $\chi^{2}(1)=4.79, p=.03$ and type of stimuli, $\chi^{2}(1)=223.75, p<.001$, were significant. Conversely, the effect of source memory score was not significant, $\chi^{2}(1)=1.49, p=.22$. Critically, the interactions semantic memory score by type of stimuli and episodic memory score by type of stimuli were found to be significant, respectively, $\chi^{2}(3)=10.83, p=.01 ; \chi^{2}(3)=24.22, p<.001$.

The significant interaction semantic memory scores by type of stimuli (Figure 2A) indicates that for critical lures the higher the semantic memory score, the higher the chances of making false memories, $z=2.19, p=.03$. No effect was found for studied words, $z=.33, p=.74$, weakly related lures, $z=$ $.16, p=.87$, nor for unrelated words, $z=-1.77, p=.08$.

The significant interaction episodic memory scores by type of stimuli (Figure 2B) indicates that for critical lures, $z=-2.33, p=.02$, weakly related lures, $z=-2.25, p=.02$, and unrelated words, $z=$ $2.62, p=.01$, the higher the episodic memory score, the lower the chances of making false memories. No effect was found for studied words, $z=.88, p=.38$. 
Conversely, the interaction source memory score by type of stimuli was not significant, $\chi^{2}(3)=.36$, $p=.94$ (Figure 2C).

A

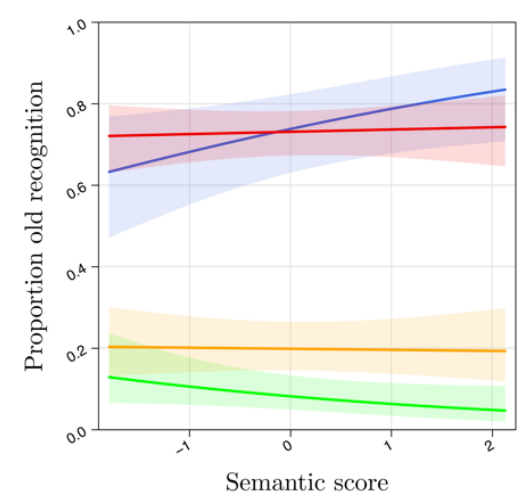

Critical lures
B

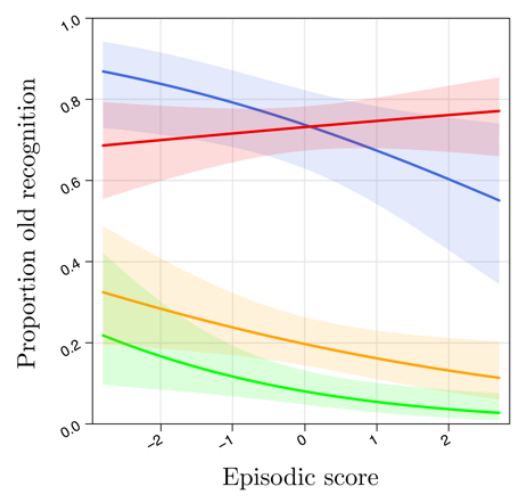

Type of stimuli

Weakly related lures Unrelated words
C

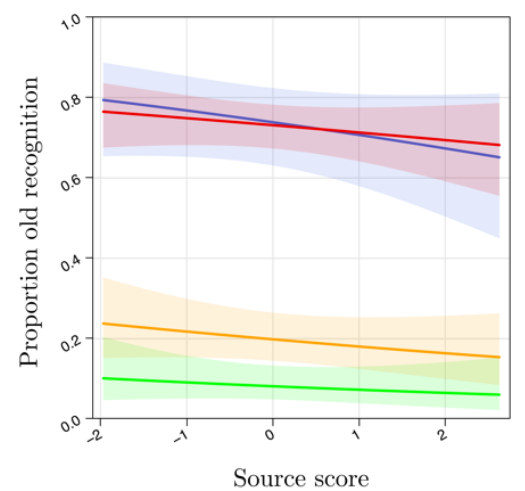

Studied words

Figure 2. Results from the GLMM including the interaction type of stimuli by semantic memory score (A), episodic memory score (B) and memory source score (C) as predictors and the proportion of "old" responses (divided in critical lures, weakly related lures, unrelated words and studied words) as dependent variable. The results show that the occurrence of false recognitions of the critical lures is predicted by both semantic and episodic memory scores, albeit with different directions (i.e., more false memories for individuals with better semantic memory abilities; less false memories for individuals with worse episodic abilities). On the contrary, the source memory score does not have any effect on false memories.

\section{Discussion}

In the present study, we investigated individual differences in memory processes subserving false recognitions in a classic DRM task. In particular, we assessed participants' semantic, episodic and source memory abilities and explored whether these three memory components differently predicted performance in the DRM task. Our findings indicate that the occurrence of false memories is predicted by both semantic and episodic memory, yet to a different extent. That is, higher semantic memory abilities predicted a larger number of false memories, while higher episodic abilities predicted a smaller number of false memories. Interestingly, the effect of semantic memory was specific for critical lures (i.e., better semantic memory abilities were associated with an increased likelihood of false recognitions), while the effect of episodic memory was found for all the three types of new stimuli, namely critical lures, weakly related lures and unrelated words (i.e., better episodic memory abilities were associated with a reduced likelihood of memory errors). 
These findings have potential implications for our understanding of false memories formation. First, previous studies investigated semantic memory involvement in the DRM task only at the item level (e.g., Roediger et al., 2001). That is, these studies manipulated the level of semantic memory involvement during the DRM task, by including word lists that could be differently associated with a critical lure (i.e., hence having lists more semantically related with critical lures, Brainerd et al., 2008). Typically, the more the word lists are semantically related with the critical lure, the higher the occurrence of false memories (Brainerd et al., 2008; Cann et al., 2011; Gatti et al., 2022a, 2022b; Roediger et al., 2001). Our findings, by showing that also differences in participants' semantic abilities play a role in the formation of false memories, indicate that these two components (i.e., the semantic content of the words processed and the extent to which participants rely on semantic memory) are possibly interacting during the DRM task. Second, by showing that also participants' episodic abilities contribute to both veridical and false memories, our data suggest that episodic memory is actively involved while judging if a word was studied or not. Such an effect is likely reflecting the recall-to-reject process (Tulving, 1983) in its diagnostic component (Gallo, 2004). That is, in the recognition phase, participants would recall the words studied and compare them with the ones actually presented: if the episodic trace for the word actually presented is weaker compared to the ones studied, the participant is less likely to accept the word as "old" (Gallo, 2004, Israel \& Schacter, 1997). Notably, the contribution of episodic processes would be hard to be operationalized at the item level, again testifying the advantages of the methodological approach employed here.

Conversely, source memory - the participants' ability to retrieve the exact perceptive features (i.e., the color of the studied words) - did not have any impact on the DRM task. These findings, indicate that source memory abilities (at least in the way we operationalized them, but for an alternative perspective on source memory see: Gallo, Korthauer, McDonough, Teshale, \& Johnson, 2011) might not play a major role in true and false memories formation. To account for this null finding we note that, in the DRM task, the studied words are typically presented from the same source (e.g., in the same ink color or read aloud by the same experimenter). In the studies in which the source of the information was manipulated (e.g., which experimenter read the study list; Payne, Elie, Blackwell, \& Neuschatz, 1996; but see also: Hicks, \& Marsh, 1999) participants certainly noticed the difference, but this did not substantially affect the rate of false memories reported. Our operationalization of source memory ability thus may tap on aspects that are not related to the specific cognitive requirements of the DRM task. Additionally, the lack of a source memory involvement in correctly rejecting new stimuli could also be specifically related to the fact that here both the encoding and recognition phases were presented in the visual modality (while a mixed auditory-visual modality has been employed in some previous research; e.g., Roediger \& McDermott, 1995). That is, the sensory 
facilitation occurring in the visual modality could have induced participants to rely on a lesser extent on source memory.

More generally, these data support the utility of individual differences for investigating the factors involved in false memory production and formation. Previous studies have indeed investigated participants' performance in the DRM task using an individual differences approach showing that several variables underly some of the individual variation in susceptibility to memory illusions. For example, participants' performance in the DRM task has been predicted from working memory abilities (Holden et al., 2020; Unsworth \& Brewer, 2010; Watson et al., 2005), age (Norman \& Schacter, 1997; Watson et al., 2001), memory self-efficacy (Iacullo et al., 2016), creativity (Dewhurst et al., 2011), or need for cognition (Graham, 2007; Leding, 2011). Here, for the first time, we employed individuals' semantic and episodic memory to predict responses in the DRM task. Our results support the specific contribution of episodic and semantic processes in the production of false memories, thus corroborating previous theoretical evidence accounting for the DRM task.

In particular, these findings directly support the two mainstream theories accounting for false memory, the AMF and FTT theories, which predict the occurrence of false recognitions on an associative/semantic basis, while adequate episodic memory processes should counter them (Brainerd, \& Reyna, 2002; Gallo \& Roediger, 2002; Reyna \& Brainerd, 1995; Roediger et al., 2001). The main difference between AMF and FTT is that the former assumes that critical lures are falsely recognized due to the associative link between that and the list words, while according to the latter theory critical lures and list words would share semantic features that underlie false recognition. Yet, disentangling the associative from the semantic framework may be sometimes difficult, as often two words that are associatively related are also semantically related (Grossman \& Eagle, 1970; Thompson-Schill, Kurtz, \& Gabrieli, 1998; but see Brainerd et al., 2008). This is also reflected in the task used here to assess semantic memory, which necessarily involves both semantic and associative processes and was selected on purpose to be representative for both AMF and FTT hypotheses.

On the one hand, according to the AMF, during the encoding phase, the presentation of the list words would associatively hyperactivate the critical lure (Gallo \& Roediger, 2002; Roediger et al., 2001). Consistent with this possibility, Roediger and colleagues (2001) have shown that the more the list words are associatively related to the critical lure, the more the participants are prone to false memories and recognitions. Crucially, the AMF hypothesis also posits that, during the recognition phase, successful monitoring processes (i.e., the ability to distinguish whether retrieved information refers to past events or not; Johnson \& Raye, 1981) can counteract false recognitions and enhance veridical memories (Gallo \& Roediger, 2002; Roediger et al., 2001). In line with this possibility, here we showed that the higher the participants' episodic memory ability, the lower the chances of making 
false memories. Critically, this effect was not limited to critical lures, but spread as well to other types of new words (i.e., weakly related lures and unrelated words), indicating that participants could adopt the same strategies in rejecting the various types of new stimuli.

On the other hand, according to the FTT participants' false memories would rely on a semantic trace, called gist trace and linked to the semantic content of each list (Brainerd, \& Reyna, 2002; Reyna \& Brainerd, 1995). Such a theoretical framework is as well in line with our findings: participants with higher semantic memory abilities are thought to have stronger abilities in forming the gist trace, resulting in turn in a higher false recognition occurrence. Furthermore, according to the FTT, correct rejections would depend on the verbatim trace, linked to both episodic and perceptive features of the studied words (Brainerd, \& Reyna, 2002; Reyna \& Brainerd, 1995). Here, however, we could only find evidence for a relationship between episodic memories and false recognitions, but not with source memory (i.e., defined in our study as the ability to correctly retrieve the perceptual features of studied words).

Beyond the two main theoretical hypotheses accounting for false memory, previous evidence suggests that false recognitions follow a continuous semantic gradient in terms of backward associative strength (Roediger et al., 2001) and semantic similarity (Gatti et al., 2022a, 2022b) between the studied words and the critical lures. Similarly, it has been shown that the semantic component that should elicit false recognitions can be decomposed into various sub-components (situation features, synonyms, antonyms, and taxonomic relations; Cann et al., 2011). The results of the present study extend these findings by showing that also individual differences in semantic and episodic memory play a crucial role in determining false memory production. These results also point out the need for investigating semantic and episodic processes while analyzing participants' performance in the DRM task in order to link false recognition proportion to episodic vs. semantic modulation. For example, employing this approach in neuromodulation studies would allow for a better comprehension of the underlying memory processes also on a neural level (i.e., if enhanced false recognition depends on impaired episodic memory on enhanced semantic memory; for alternative approaches see also: Diez, Gomez-Ariza, Diez-Alamo, Alonso, \& Fernandez, 2017; Gatti, Vecchi, \& Mazzoni, 2021).

Finally, one possible point of concern could be related to the lack of effects of semantic and episodic memory on veridical recognition. To account for this pattern of results, we first note that, besides unrelated words, we also used as control stimuli weakly related words; these weakly related words could have induced a shift in participants' response criterion favoring in certain cases a conservative response bias (i.e., recognizing that also weakly related words were shown in the recognition task could have induced some participants to respond "no" more often to less semantically related studied 
words). Second, as the semantic features of the studied words underlying false recognitions comprise several sub-components (Cann et al., 2011), participants' semantic memory could be decomposed into different sub-systems involved in veridical and false recognitions. Consequently, extracting the semantic score from another task could better account for veridical recognition, with this approach being particularly promising for future research in order to distinguish between the AMF and FTT theories.

In conclusion, by taking an individual differences approach, in this study we show that various memory systems are differently involved in veridical and false memories production. Our findings thus point to the importance of investigating individual differences linked to false memory production, possibly through more ecological paradigms, since this has ultimately links with the quality of justice trials and witness evaluation (Schacter \& Loftus, 2013). 


\section{Declarations}

\section{Author Contributions}

DG: Conceptualization, Methodology, Formal analysis, Writing - Original draft preparation. LR: Conceptualization, Methodology, Formal analysis, Writing - Original draft preparation. GM and TV: Conceptualization, Methodology, Writing - Review \& editing, Supervision. All authors approved the final version of the manuscript for submission.

\section{Conflicts of interest}

The authors declare no conflicts of interest.

\section{Funding}

This work was supported by funding from the Italian Ministry of University and Research (PRIN 2017 no. 201755TKFE) to TV and from Italian Ministry of Health (Ricerca Corrente 2021) to LR and TV.

\section{Data availability}

The data used in this study are reported as Supplementary Materials.

\section{References}

Argyropoulos, G.P., \& Muggleton, N.G. (2013). Effects of cerebellar stimulation on processing semantic associations. The Cerebellum, 12(1), 83-96.

Baddeley, A., Eysenck, M.W., \& Anderson, M.C. (2009). Memory. Psychology Press.

Balota, D.A., Cortese, M.J., Duchek, J.M., Adams, D., Roediger, H.L., McDermott, K.B., \& Yerys, B.E. (1999). Veridical and false memories in healthy older adults and in dementia of the Alzheimer's type. Cognitive Neuropsychology, 16(3-5), 361-384.

Baroni, M., Dinu, G., \& Kruszewski, G. (2014). Don't count, predict! A systematic comparison of context-counting vs. context-predicting semantic vectors. In K. Toutanova \& H. Wu (Eds.), Proceedings of the 52nd Annual Meeting of the Association for Computational Linguistics (Vol. 1, pp. 238-247). Stroudsburg, PA: Association for Computational Linguistics.

Bartlett, F.C. (1932). Remembering. Cambridge University Press.

Bates, D., Maechler, M., Bolker, B., \& Walker, S. (2015). Fitting Linear Mixed-Effects Models Using lme4. Journal of Statistical Software, 67(1), 1-48. 
Brainerd, C.J., \& Reyna, V.F. (2002). Fuzzy-trace theory and false memory. Current Directions in Psychological Science, 11(5), 164-169.

Brainerd, C.J., Yang, Y., Reyna, V.F., Howe, M.L., \& Mills, B.A. (2008). Semantic processing in “associative” false memory. Psychonomic Bulletin \& Review, 15(6), 1035-1053.

Cacioppo, J.T., \& Petty, R.E. (1982). The need for cognition. Journal of Personality and Social Psychology, 42, 116-131.

Cann, D.R., McRae, K., \& Katz, A.N. (2011). False recall in the Deese-Roediger-McDermott paradigm: The roles of gist and associative strength. Quarterly Journal of Experimental Psychology, 64(8), 1515-1542.

Chen, N.F., Lo, C.M., Liu, T.L., \& Cheng, S.K. (2016). Source memory performance is modulated by transcranial direct current stimulation over the left posterior parietal cortex. NeuroImage, $139,462-469$.

Christensen, R.H.B. (2019). ordinal - Regression Models for Ordinal Data. R package version 2019.12-10. https://CRAN.R-project.org/package=ordinal.

Deese, J. (1959). On the prediction of occurrence of particular verbal intrusions in immediate recall. Journal of Experimental Psychology, 58(1), 17-22.

Della Rosa, P.A., Catricalà, E., Vigliocco, G., \& Cappa, S.F. (2010). Beyond the abstract-concrete dichotomy: mode of acquisition, concreteness, imageability, familiarity, age of acquisition, context availability, and abstractness norms for a set of 417 Italian words. Behavior Research Methods, 42, 1042-1048.

Dewhurst, S.A., Thorley, C., Hammond, E.R., \& Ormerod, T.C. (2011). Convergent, but not divergent, thinking predicts susceptibility to associative memory illusions. Personality and Individual Differences, 51(1), 73-76.

Diez, E., Gomez-Ariza, C.J., Diez-Alamo, A.M., Alonso, M.A., \& Fernandez, A. (2017). The processing of semantic relatedness in the brain: Evidence from associative and categorical false recognition effects following transcranial direct current stimulation of the left anterior temporal lobe. Cortex, 93, 133-145.

Fox, J. (2003). Effect Displays in R for Generalised Linear Models. Journal of Statistical Software, 8(15), 1-27. URL http://www.jstatsoft.org/v08/i15/.

Fox, J., \& Weisberg, S. (2019). An R Companion to Applied Regression, 3rd Edition. Thousand Oaks, $\mathrm{CA}<\mathrm{http}: / /$ tinyurl.com/carbook $>$

Gallo, D.A. (2010). False memories and fantastic beliefs: 15 years of the DRM illusion. Memory \& Cognition, 38(7), 833-848. 
Gallo, D.A. (2004). Using recall to reduce false recognition: diagnostic and disqualifying monitoring. Journal of Experimental Psychology: Learning, Memory, and Cognition, 30(1), 120.

Gallo, D.A., Korthauer, L.E., McDonough, I.M., Teshale, S., \& Johnson, E.L. (2011). Age-related positivity effects and autobiographical memory detail: Evidence from a past/future source memory task. Memory, 19(6), 641-652.

Gallo, D.A., \& Roediger, H.L. (2002). Variability among word lists in eliciting memory illusions: Evidence for associative activation and monitoring. Journal of Memory and Language, 47(3), 469-497.

Gatti, D., Rinaldi, L., Marelli, M., Mazzoni, G., \& Vecchi, T. (2022a). Decomposing the semantic processes underpinning veridical and false memories. Journal of Experimental Psychology: General, 151(2), 363-389.

Gatti, D., Marelli, M., Mazzoni, G., Vecchi, T., \& Rinaldi, L. (2022b). Hands on false memory: a mouse-tracking study with the DRM task. Psychological Research.

Gatti, D., Marelli, M., \& Rinaldi, L. (in press). Out-of-vocabulary but not meaningless: Evidence for semantic-priming effects in pseudoword processing. Journal of Experimental Psychology: General.

Gatti, D., Vecchi, T., \& Mazzoni, G. (2021). Cerebellum and semantic memory: a TMS study using the DRM paradigm. Cortex.

Graham, L.M. (2007). Need for cognition and false memory in the Deese-Roediger-McDermott paradigm. Personality and Individual Differences, 42, 409-418.

Grossman, L., \& Eagle, M. (1970). Synonymity, antonymity, and association in false recognition responses. Journal of Experimental Psychology, 83(2p1), 244

Günther, F., Rinaldi, L., \& Marelli, M. (2019). Vector-space models of semantic representation from a cognitive perspective: A discussion of common misconceptions. Perspectives on Psychological Science, 14(6), 1006-1033.

Hicks, J.L., \& Marsh, R.L. (1999). Attempts to reduce the incidence of false recall with source monitoring. Journal of Experimental Psychology: Learning, Memory, and Cognition, 25(5), 1195.

Holden, L.R., Conway, A.R.A., \& Goodwin, K.A. (2020). How Individual Differences in Working Memory and Source Monitoring matter in Susceptibility to False Memory. https://doi.org/10.31234/osf.io/h48bv

Hutchison, K.A., Balota, D.A., Neely, J.H., Cortese, M.J., Cohen-Shikora, E. R., Tse, C.S., ... \& Buchanan, E. (2013). The semantic priming project. Behavior Research Methods, 45(4), $1099-1114$ 
Iacullo, V.M., \& Marucci, F.S. (2016). Normative data for Italian Deese/Roediger-McDermott lists. Behavior Research Methods, 48(1), 381-389.

Iacullo, V.M., Marucci, F.S., \& Mazzoni, G. (2016). Inducing false memories by manipulating memory self-efficacy. Learning and Individual Differences, 49, 237-244.

Israel, L., \& Schacter, D.L. (1997). Pictorial encoding reduces false recognition of semantic associates. Psychonomic Bulletin \& Review, 4(4), 577-581.

Johnson, M.K., \& Raye, C.L. (1981). Reality monitoring. Psychological Review, 88(1), 67.

Koerner, T.K., \& Zhang, Y. (2017). Application of linear mixed-effects models in human neuroscience research: a comparison with Pearson correlation in two auditory electrophysiology studies. Brain Sciences, 7(3), 26.

Leding, J.K. (2011). Need for cognition and false recall. Personality and Individual Differences, 51(1), 68-72.

Makowski, D. (2018). The psycho Package: an Efficient and Publishing-Oriented Workflow for Psychological Science. Journal of Open Source Software, 3(22), 470.

Mandera, P., Keuleers, E., \& Brysbaert, M. (2017). Explaining human performance in psycholinguistic tasks with models of meaning distance based on prediction and counting: A review and empirical validation. Journal of Memory and Language, 92, 57-78.

Marelli, M. (2017). Word-embeddings Italian Semantic Spaces: A semantic model for psycholinguistic research. Psihologija, 50(4), 503-520.

Montefinese, M., Ambrosini, E., Fairfield, B., \& Mammarella, N. (2013). Semantic memory: A feature-based analysis and new norms for Italian. Behavior Research Methods, 45(2), 440461.

Norman, K.A., \& Schacter, D.L. (1997). False recognition in younger and older adults: Exploring the characteristics of illusory memories. Memory \& Cognition, 25, 838-848.

Payne, D.G., Elie, C.J., Blackwell, J.M., \& Neuschatz, J.S. (1996). Memory illusions: Recalling, recognizing, and recollecting events that never occurred. Journal of Memory and Language, $35(2), 261-285$.

Pansuwan, T., Breuer, F., Gazder, T., Lau, Z., Cueva, S., Swanson, L., ... \& Morcom, A. M. (2020). Evidence for adult age-invariance in associative false recognition. Memory, 28(2), 172-186.

Peirce, J.W. (2007). PsychoPy - Psychophysics software in Python. Journal of Neuroscience Methods, 162 (1-2), 8-13.

Peirce, J.W. (2009). Generating stimuli for neuroscience using PsychoPy. Frontiers in Neuroinformatics, 2 (10), 1-8.

Peirce, J.W., \& MacAskill, M.R. (2018). Building Experiments in PsychoPy. London: Sage. 
Peirce, J.W., Gray, J.R., Simpson, S., MacAskill, M.R., Höchenberger, R., Sogo, H., Kastman, E., Lindeløv, J. (2019). PsychoPy2: experiments in behavior made easy. Behavior Research Methods.

Perea, M., \& Gotor, A. (1997). Associative and semantic priming effects occur at very short stimulusonset asynchronies in lexical decision and naming. Cognition, 62(2), 223-240.

Reyna, V.F., \& Brainerd, C.J. (1995). Fuzzy-trace theory: An interim synthesis. Learning and Individual Differences, 7(1), 1-75.

Roediger, H.L., \& McDermott, K.B. (1995). Creating False Memories: Remembering Words Not Presented in Lists. Journal of Experimental Psychology: General, 21(4), 803-814.

Roediger, H.L., Watson, J.M., McDermott, K.B., \& Gallo, D.A. (2001). Factors that determine false recall: A multiple regression analysis. Psychonomic Bulletin \& Review, 8(3), 385-407.

RStudio Team (2015). RStudio: Integrated Development for R. RStudio, Inc., Boston, MA URL http://www.rstudio.com/.

Schacter, D.L. (2001). The seven sins of memory: How the mind forgets and remembers. Mifflin and Company.

Schacter, D.L., \& Loftus, E.F. (2013). Memory and law: what can cognitive neuroscience contribute?. Nature Neuroscience, 16(2), 119-123.

Seamon, J.G., Luo, C.R., \& Gallo, D.A. (1998). Creating false memories of words with or without recognition of list items: Evidence for nonconscious processes. Psychological Science, 9(1), 20-26.

Stanislaw, H., \& Todorov, N. (1999). Calculation of signal detection theory measures. Behavior Research Methods, Instruments, \& Computers, 31(1), 137-149.

Sulin, R.A., \& Dooling, D.J. (1974). Intrusion of a Thematic Idea in Retention of Prose. Journal of Experimental Psychology, 103(2), 255-262.

Thompson-Schill, S.L., Kurtz, K.J., \& Gabrieli, J.D. (1998). Effects of semantic and associative relatedness on automatic priming. Journal of Memory and Language, 38(4), 440-458.

Tulving, E. (1983). Elements of episodic memory. London: Oxford University Press.

Tun, P.A., Wingfield, A., Rosen, M.J., \& Blanchard, L. (1998). Response latencies for false memories: gist-based processes in normal aging. Psychology and Aging, 13(2), 230.

Unsworth, N., \& Brewer, G.A. (2010). Individual differences in false recall: A latent variable analysis. Journal of Memory and Language, 62, 19-34

Vecchi, T. \& Gatti, D. (2020). Memory as prediction: From looking back to looking forward. MIT Press. 
Watson, J.M., Balota, D.A., \& Sergent-Marshall, S. D. (2001). Semantic, phonological, and hybrid veridical and false memories in healthy older adults and in individuals with dementia of the Alzheimer Type. Neuropsychology, 15, 254-267.

Watson, J.M., Bunting, M.F., Poole, B.J., \& Conway, A.R. (2005). Individual differences in susceptibility to false memory in the Deese-Roediger-McDermott paradigm. Journal of Experimental Psychology: Learning, Memory, and Cognition, 31(1), 76.

Westerberg, C. E., \& Marsolek, C. J. (2006). Do instructional warnings reduce false recognition?. Applied Cognitive Psychology, 20(1), 97-114. 